\title{
A Review of Signal Subspace Speech Enhancement and Its Application to Noise Robust Speech Recognition
}

\author{
Kris Hermus, Patrick Wambacq, and Hugo Van hamme \\ Department of Electrical Engineering - ESAT, Katholieke Universiteit Leuven, 3001 Leuven-Heverlee, Belgium
}

Received 24 October 2005; Revised 7 March 2006; Accepted 30 April 2006

Recommended by Kostas Berberidis

\begin{abstract}
The objective of this paper is threefold: (1) to provide an extensive review of signal subspace speech enhancement, (2) to derive an upper bound for the performance of these techniques, and (3) to present a comprehensive study of the potential of subspace filtering to increase the robustness of automatic speech recognisers against stationary additive noise distortions. Subspace filtering methods are based on the orthogonal decomposition of the noisy speech observation space into a signal subspace and a noise subspace. This decomposition is possible under the assumption of a low-rank model for speech, and on the availability of an estimate of the noise correlation matrix. We present an extensive overview of the available estimators, and derive a theoretical estimator to experimentally assess an upper bound to the performance that can be achieved by any subspace-based method. Automatic speech recognition (ASR) experiments with noisy data demonstrate that subspace-based speech enhancement can significantly increase the robustness of these systems in additive coloured noise environments. Optimal performance is obtained only if no explicit rank reduction of the noisy Hankel matrix is performed. Although this strategy might increase the level of the residual noise, it reduces the risk of removing essential signal information for the recogniser's back end. Finally, it is also shown that subspace filtering compares favourably to the well-known spectral subtraction technique.
\end{abstract}

Copyright (c) 2007 Kris Hermus et al. This is an open access article distributed under the Creative Commons Attribution License, which permits unrestricted use, distribution, and reproduction in any medium, provided the original work is properly cited.

\section{INTRODUCTION}

One particular class of speech enhancement techniques that has gained a lot of attention is signal subspace filtering. In this approach, a nonparametric linear estimate of the unknown clean-speech signal is obtained based on a decomposition of the observed noisy signal into mutually orthogonal signal and noise subspaces. This decomposition is possible under the assumption of a low-rank linear model for speech and an uncorrelated additive (white) noise interference. Under these conditions, the energy of less correlated noise spreads over the whole observation space while the energy of the correlated speech components is concentrated in a subspace thereof. Also, the signal subspace can be recovered consistently from the noisy data. Generally speaking, noise reduction is obtained by nulling the noise subspace and by removing the noise contribution in the signal subspace.

The idea to perform subspace-based signal estimation was originally proposed by Tufts et al. [1]. In their work, the signal estimation is actually based on a modified SVD of data matrices. Later on, Cadzow [2] presented a general framework for recovering signals from noisy observations. It is assumed that the original signal exhibits some well-defined properties or obeys a certain model. Signal enhancement is then obtained by mapping the observed signal onto the space of signals that possess the same structure as the clean signal. This theory forms the basis for all subspace-based noise reduction algorithms.

A first and indispensable step towards noise reduction is obtained by nulling the noise subspace (least squares (LS) estimator) [3]. However, for improved noise reduction, also the noise contribution in the (signal + noise) subspace should be suppressed or controlled, which is achieved by all other estimators as is explained in subsequent sections of this paper.

Of particular interest is the minimum variance (MV) estimation, which gives the best linear estimate of the clean data, given the rank $p$ of the clean signal and the variance of the white noise $[4,5]$. Later on, a subspace-based speech enhancement with noise shaping was proposed in [6]. Based on the observation that signal distortion and residual noise cannot be minimised simultaneously, two new linear estimators 
are designed-time domain constrained (TDC) and spectral domain constrained (SDC) - that keep the level of the residual noise below a chosen threshold while minimising signal distortion. Parameters of the algorithm control the trade-off between residual noise and signal distortion. In subspacebased speech enhancement with true perceptual noise shaping, the residual noise is shaped according to an estimate of the clean signal masking threshold, as discussed in more recent papers [7-9].

Although basic subspace-based speech enhancement is developed for dealing with white noise distortions, it can easily be extended to remove general coloured noise provided that the noise covariance matrix is known (or can be estimated) $[10,11]$. A detailed theoretical analysis of the underlying principles of subspace filtering can, for example, be found in $[4,6,12]$.

The excellent noise reduction capabilities of subspace filtering techniques are confirmed by several studies, both with the basic LS estimate [3] and with the more advanced optimisation criteria $[6,10,13]$. Especially for the MV and SDC estimators, a speech quality improvement that outperforms the spectral subtraction approach is revealed by listening tests.

Noise suppression facilitates the understanding, communication, and processing of speech signals. As such, it also plays an important role in automatic speech recognition (ASR) to improve the robustness in noisy environments. The latter is achieved by enhancing the observed noisy speech signal prior to the recogniser's preprocessing and decoding operations. In ASR applications, the effectiveness of any speech enhancement algorithm is quantified by its potential to close the gap between noisy and clean-speech recognition accuracy.

Opposite to what happens in speech communication applications, the improvement in intelligibility of the speech and the reduction of listener's fatigue are of no concern. Nevertheless, a correlation can be expected between the improvements in perceived speech quality on the one hand, and the improvement in recognition accuracy on the other hand.

Very few papers discuss the application of signal subspace methods to robust speech recognition. In [14] an energy-constrained signal subspace (ECSS) method is proposed based on the MV estimator. For the recognition of large-vocabulary continuous speech (LV-CS) corrupted by additive white noise, a relative reduction in WER of $70 \%$ is reported. In [15], MV subspace filtering is applied on a LV-CS recognition (LV-CSR) task distorted with white and coloured noise. Significant WER reductions that outperform spectral subtraction are reported.

\section{Paper outline}

In this paper we elaborate on previous paper [16] and describe the potential of subspace-based speech enhancement to improve the performance of ASR in noisy conditions. At first, we extensively review several subspace estimation techniques and classify these techniques based on the optimisation criteria. Next, we conduct a performance comparison for both white and coloured noise removal from a speech enhancement and especially from a speech recognition perspective. The impact of some crucial parameters, such as the analysis window length, the Hankel matrix dimensions, the signal subspace dimension, and methodspecific design parameters will be discussed.

\section{SUBSPACE FILTERING}

\subsection{Fundamentals}

Any noise reduction technique requires assumptions about the nature of the interfering noise signal. Subspace-based speech enhancement also makes some basic assumptions about the properties of the desired signal (clean speech) as is the case in many-but not all-signal enhancement algorithms. Evidently, the separation of the speech and noise signals will be based on their different characteristics.

Since the characteristics of the speech (and also of the noise) signal(s) are time varying, the speech enhancement procedure is performed on overlapping analysis frames.

\section{Speech signal}

A key assumption in all subspace-based signal enhancement algorithms is that every short-time speech vector $s=$ $[s(1), s(2), \ldots, s(q)]^{T}$ can be written as a linear combination of $p<q$ linearly independent basis functions $m_{i}, i=1, \ldots, p$,

$$
s=M y
$$

where $M$ is a $(q \times p)$ matrix containing the basis functions (column-wise ordered) and $y$ is a length- $p$ column vector containing the weights. Both the number and the form of these basis functions will in general be time varying (framedependent).

An obvious choice for $m_{i}$ are (damped) sinusoids motivated by the traditional sinusoidal model (SM) for speech signals. A crucial observation here is that the consecutive speech vectors $s$ will occupy a $(p<q)$-dimensional subspace of the $q$-dimensional Euclidean space ( $p$ equals the signal order). Because of the time-varying nature of speech signals, the location of this signal subspace (and its dimension) will consequently be frame-dependent.

\section{Noise signal}

The additive noise is assumed to be zero-mean, white, and uncorrelated with the speech signal. Its variance should be slowly time varying such that it can be estimated from noiseonly segments. Contrarily to the speech signal, consecutive noise vectors $n$ will occupy the whole q-dimensional space.

\section{Speech/noise separation}

Based on the above description of the speech and noise signals, the aforementioned $q$-dimensional observation space is split in two subspaces, namely a $p$-dimensional (signal + noise) subspace in which the noise interferes with the speech signal, and a $(q-p)$-dimensional subspace that contains only 
noise (and no speech). The speech enhancement procedure can now be summarised as follows:

(1) separate the (signal+noise) subspaces from the (noiseonly) subspace,

(2) remove the (noise-only) subspace,

(3) optionally, remove the noise components in the (signal + noise) subspace. ${ }^{1}$

The first operation is straightforward for the white noise condition under consideration here, but can become complicated for the coloured noise case as we will see further on. The second operation is applied in all implementations of subspace-based signal enhancements, whereas the third operation is indispensable to obtain an increased noise reduction. Nevertheless, the last operation is sometimes omitted because of the introduction of speech distortion. The latter problem is inevitable since the speech and noise signals overlap in the signal subspace.

In the next section we will explain that the orthogonal decomposition into frame-dependent signal and noise subspaces can be performed by an SVD of the noisy signal observation matrix, or equivalently by an eigenvalue decomposition (EVD) of the noisy signal correlation matrix.

\subsection{Algorithm}

Let $s(k)$ represent the clean-speech samples and let $n(k)$ be the zero-mean, additive white noise distortion that is assumed to be uncorrelated with the clean speech. The observed noisy speech $x(k)$ is then given by

$$
x(k)=s(k)+n(k) .
$$

Further, let $\bar{R}_{x}, \bar{R}_{s}$, and $\bar{R}_{n}$ be $(q \times q)$ (with $\left.q>p\right)$ true autocorrelation matrices of $x(k), s(k)$, and $n(k)$, respectively. Due to the assumption of uncorrelated speech and noise, it is clear that

$$
\bar{R}_{x}=\bar{R}_{s}+\bar{R}_{n}
$$

The EVD of $\bar{R}_{s}, \bar{R}_{n}$, and $\bar{R}_{x}$ can be written as follows:

$$
\begin{aligned}
& \bar{R}_{s}=\bar{V} \bar{\Lambda} \bar{V}^{T}, \\
& \bar{R}_{n}=\bar{V}\left(\sigma_{w}^{2} I\right) \bar{V}^{T}, \\
& \bar{R}_{x}=\bar{V}\left(\bar{\Lambda}+\sigma_{w}^{2} I\right) \bar{V}^{T},
\end{aligned}
$$

with $\bar{\Lambda}$ a diagonal matrix containing the eigenvalues $\bar{\lambda}_{i}, \bar{V}$ an orthonormal matrix containing the eigenvectors $\bar{v}_{i}, \sigma_{w}^{2}$ the noise variance, and $I$ the identity matrix. A crucial observation here is that the eigenvectors of the noise are identical to the clean-speech eigenvectors due to the white noise assumption such that the eigenvectors of $\bar{R}_{s}$ can be found from the EVD of $\bar{R}_{x}$ in (6).

\footnotetext{
${ }^{1}$ For brevity, the (signal + noise) subspace will further be called the signal subspace, and the (noise-only) subspace will be referred to as the noise subspace.
}

Based on the assumption that the clean speech is confined to a $(p<q)$-dimensional subspace $(1)$, we know that $\bar{R}_{s}$ has only $p$ nonzero eigenvalues $\bar{\lambda}_{i}$. If

$$
\bar{\lambda}_{i}>\sigma_{w}^{2} \quad(i=1, \ldots, p),
$$

the noise can be separated from the speech signal, and the EVD of $\bar{R}_{x}$ can be rewritten as

$$
\left.\bar{R}_{x}=\left[\bar{V}_{p} \bar{V}_{q-p}\right]\left(\left[\begin{array}{cc}
\bar{\Lambda}_{p} & 0 \\
0 & 0
\end{array}\right]+\sigma_{w}^{2}\left[\begin{array}{cc}
I_{p} & 0 \\
0 & I_{q-p}
\end{array}\right]\right)\left[\begin{array}{cc}
\bar{V}_{p} & \bar{V}_{q-p}
\end{array}\right]^{T}\right]
$$

if we assume that the elements $\bar{\lambda}_{i}$ of $\bar{\Lambda}$ are in descending order. The subscripts $p$ and $q-p$ refer to the signal and noise subspaces, respectively.

Regardless of the specific optimisation criterion, speech enhancement is now obtained by

(1) restricting the enhanced speech to occupy solely the signal subspace by nulling its components in the noise subspace,

(2) changing (i.e., lowering) the eigenvalues that correspond to the signal subspace.

Mathematically this enhancement procedure can be written as a filtering operation on the noisy speech vector $x=$ $[x(1), x(2), \ldots, x(q)]^{T}$ :

$$
\widehat{s}=F x
$$

with the filter matrix $F$ given by

$$
F=\bar{V}_{p} G_{p} \bar{V}_{p}^{T}
$$

in which the $(p \times p)$ diagonal matrix $G_{p}$ contains the weighting factors $g_{i}$ for the first $p$ eigenvalues of $\bar{R}_{x}$, while $\bar{V}^{T}$ and $\bar{V}$ are known as the KLT (Karhunen Loeve transform) matrix and its inverse, respectively. The filter matrix $F$ can be rewritten as

$$
F=\sum_{i=1}^{p} g_{i} \bar{v}_{i} \bar{v}_{i}^{T}
$$

which illustrates that the filtered signal can be seen as the sum of $p$ outputs of a "filter bank" (see below). Each filter in this filter bank is solely dependent on one eigenvector $\bar{v}_{i}$ and its corresponding gain factor $g_{i}$.

\section{From EVD to SVD filtering}

In many implementations the true covariance matrices in (4) to (6) are estimated as $R_{x}=H_{x}^{T} H_{x}$, with $H_{x}\left(=H_{s}+H_{n}\right)$ an $(m \times q)$ (with $m>q)$ noisy Hankel (or Toeplitz) ${ }^{2}$ signal observation matrix constructed from a noisy speech vector $x$

\footnotetext{
${ }^{2}$ Because of the equivalence of the Hankel and Toeplitz matrices, that is, a Toeplitz matrix can be converted into a Hankel matrix by a simple permutation of its rows, any further derivation and discussion will be restricted to Hankel matrices only.
} 


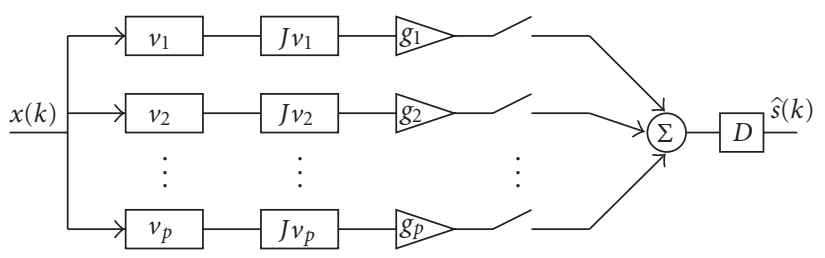

FIgURE 1: FIR-filter implementation of subspace-based speech enhancement. Each singular triplet corresponds to a zero-phase filtered version of the noisy signal.

containing $N(N \gg q$, and $m+q=N+1)$ samples of $x(k)$. In that case an equivalent speech enhancement can be obtained via the SVD of $H_{x}$ [6]. A commonly used modified SVD-based speech enhancement procedure proceeds as follows.

Let the SVD of $H_{x}$ be given by

$$
H_{x}=U \Sigma V^{T} .
$$

If the short-time speech and noise signals are orthogonal $\left(H_{s}^{T} H_{n}=0\right)$ and if the short-time noise signal is white $\left(H_{n}^{T} H_{n}=\sigma_{v}^{2} I\right)$, then

$$
H_{x}=U\left(\sqrt{\bar{\Sigma}^{2}+\sigma_{\nu}^{2} I}\right) V^{T}
$$

with $\bar{\Sigma}$ the matrix containing the singular values of the clean Hankel matrix $H_{s}$, and $\sigma_{\nu}$ the 2-norm of the columns of $H_{n}$ (observe that for large $N$ and in the case of stationary white noise, $\sigma_{v}^{2} / m$ converges in the mean square sense to $\left.\sigma_{w}^{2}\right)$.

Under weak conditions, the empirical covariance matrix $H_{x}^{T} H_{x} / N$ will converge to the true autocorrelation matrix $\bar{R}_{x}$. In other words, for sufficiently large $N$, the subspace that is spanned by the $p$ dominant eigenvectors of $V$ will converge to the subspace that is spanned by the vectors of $\bar{V}_{p}$ from (6).

The enhanced matrix $\hat{H}_{s}$ is then obtained as

$$
\hat{H}_{s}=U_{p} G_{p} \Sigma_{p} V_{p}^{T}
$$

or

$$
\hat{H}_{s}=\sum_{i=1}^{p} g_{i} \sigma_{i} u_{i} v_{i}^{T}
$$

with $\sigma_{i}$ denoting the $i$ th singular value of $\Sigma$.

The enhanced signal $\hat{s}(k)$ is recovered by averaging along the antidiagonals of $\hat{H}_{s}$. Dologlou and Carayannis [17], and later on Hansen and Jensen [18] proved that this overall procedure is equivalent to one global FIR-filtering operation on the noisy time signal (Figure 1). Each filter bank output $g_{i} \sigma_{i} u_{i} v_{i}^{T}$ is obtained by filtering the noisy signal $x(k)$ with its corresponding eigenfilter $v_{i}$ and its reversed version $J v_{i}$. From filter theory we know that this results in a zero-phase filtering operation. The extraction of the enhanced signal $\hat{s}(k)$ from the enhanced observation matrix $\hat{H}_{s}$ is equivalent to a multiplication of $\hat{H}_{s}$ by the diagonal matrix $D$ (see Figure 1). The elements
$\{1,1 / 2,1 / 3, \ldots, 1 / q, 1 / q, \ldots, 1 / q, \ldots, 1 / 3,1 / 2,1\}$ on the diagonal of $D$ account for the difference in length of the antidiagonals of the signal observation matrix.

This FIR-filter equivalence is an important finding and gives an interesting frequency-domain interpretation of the signal subspace denoising operation.

The main advantage of working with the SVD, instead of the EVD, is that no explicit estimation of the covariance matrix is needed. In this paper we will further focus on the SVD description. However, it is stressed that all estimators can as well be performed in an EVD-based scheme, which allows for the use of any arbitrary (structured) covariance estimates like, for example, the empirical Toeplitz covariance matrix.

\subsection{Optimisation criteria}

By applying a specific estimation criterion, the elements of the weighting matrix $G_{p}$ from (14) can be found. In this section the most common of these criteria are briefly reviewed. Note that the derivations and statements below are only exact if the aforementioned conditions (speech of order $p$, white noise interference, and orthogonality of speech and noise) are fulfilled.

\section{Least squares}

The least squares (LS) estimate $\hat{H}_{\text {LS }}$ is defined as the best rank- $p$ approximation of $H_{x}$ :

$$
\min _{r k\left(\hat{H}_{\mathrm{LS}}\right)=p}\left\|H_{x}-\hat{H}_{\mathrm{LS}}\right\|_{F}^{2}
$$

with $r k(A)$ and $\|A\|_{F}^{2}$ denoting the rank and the Frobenius of matrix $A$, respectively.

The LS estimate is obtained by truncating the SVD $U \Sigma V^{T}$ of $H_{x}$ to rank $p$ :

$$
\hat{H}_{\mathrm{LS}}=U_{p} \Sigma_{p} V_{p}^{T}
$$

Observe that this estimate removes the noise subspace, but keeps the noisy signal unaltered in the signal subspace. This estimate yields an enhanced signal with the highest residual noise level $\left(=(p / q) \sigma_{v}^{2}\right)$ but with the lowest signal distortion $(=0)$. The performance of the LS estimator is crucially dependent on the estimation of the signal rank $p$.

\section{Minimum variance}

Given the rank $p$ of the clean speech, the MV estimate $\hat{H}_{\mathrm{MV}}$ is the best approximation of the original matrix $H_{s}$ that can be obtained by making linear combinations of the columns of $H_{x}$ :

$$
\hat{H}_{\mathrm{MV}}=H_{x} T
$$

with

$$
T=\underset{T \in \mathbb{R}^{q \times q}}{\arg \min }\left\|H_{x} T-H_{s}\right\|_{F}^{2} .
$$


In algebraic terms, $\hat{H}_{\mathrm{MV}}$ is the geometric projection of $H_{s}$ onto the column space of $H_{x}$, and is obtained by setting

$$
g_{\mathrm{MV}, i}=1-\frac{\sigma_{v}^{2}}{\sigma_{i}^{2}} .
$$

The MV estimate is the linear estimator with the lowest residual noise level (LMMSE estimator) $[4,5]$, and is related to Wiener filtering and spectral subtraction.

\section{Singular value adaptation}

In the singular value adaptation (SVA) method [5], the $p$ dominant singular values of $H_{x}$ are mapped onto the original (clean) singular values of $H_{s}$ by setting

$$
g_{\mathrm{SVA}, i}=\frac{\sqrt{\sigma_{i}^{2}-\sigma_{\nu}^{2}}}{\sigma_{i}} .
$$

Observe that

$$
g_{\mathrm{SVA}, i}=\sqrt{g_{\mathrm{MV}, i}}
$$

which illustrates the conservative noise reduction of the SVA estimator.

\section{Time domain constrained}

The TDC estimate is found by minimising the signal distortion while setting a user-defined upper bound on the residual noise level via a control parameter $\mu \geq 0$. In the modified SVD of $H_{x}, g_{\mathrm{TDC}, i}$ is given by

$$
g_{\mathrm{TDC}, i}=\frac{1-\sigma_{\nu}^{2} / \sigma_{i}^{2}}{1-\left(\sigma_{\nu}^{2} / \sigma_{i}^{2}\right)(1-\mu)} .
$$

This estimator can be seen as a Wiener filter with adjustable input noise level $\mu \sigma_{v}^{2}[6]$.

If $\mu=0$, the gains for the signal subspace components are all set to one which means that the TDC estimator becomes equal to the LS estimator. Also, the MV estimator is a special case of TDC with $\mu=1$.

The most straightforward way to specify the value of $\mu$ is to assign a constant value to it, independently of the speech frame at hand. A more complex method is to let $\mu$ depend on the SNR of the actual frame [19]. Typically $\mu$ ranges from 2 to 3 .

\section{Spectral domain constrained}

A simple form of residual noise shaping is provided by the SDC estimator. Here, the estimate is found by minimising the signal distortion subject to constraints on the energy of the projections of the residual noise onto the signal subspace. More than one solution for the gain factors in the modified SVD exists. One possible expression for $g_{\mathrm{SDC}, i}$ is [6]

$$
g_{\mathrm{SDC}_{-} 1, i}=\sqrt{\exp \left(\frac{-\beta \sigma_{\nu}^{2}}{\sigma_{i}^{2}-\sigma_{\nu}^{2}}\right)}
$$

with $\beta \geq 0$, but mostly $\geq 1$ for sufficient noise reduction. We will further refer to this estimator as SDC_1. An alternative solution [6] is to choose

$$
g_{\mathrm{SDC} \_, i}=\left(1-\frac{\sigma_{v}^{2}}{\sigma_{i}^{2}}\right)^{\gamma / 2}
$$

with $\gamma \geq 1$, further denoted as SDC _2. The amount of noise reduction can be controlled by the parameters $\beta$ and $\gamma$. Note that the SDC $\_2$ estimator is a generalisation of both the MV estimator (20) for $\gamma=2$ and the SVA estimator (21) for $\gamma=$ 1 .

Extensions of the SDC estimator that exploit the information obtained from a perceptual model have been presented $[7,8]$.

\section{Optimal estimator}

In practice, the assumption of a low-rank speech model (1) will almost never be (exactly) met. Also, the processing of short frames will cause deviations from assumed properties such as orthogonality of speech and noise (finite sample behaviour). Consequently, the eigenvectors of the noisy speech are not identical to the clean-speech eigenvectors such that the signal subspace will not be exactly recovered $((6)$ is not valid). Also, the measurement of the perturbation of the singular values of $H_{s}$ as stated in (13) will not be exact (the singular value spectrum of the noise Hankel matrix $H_{n}$ will not be isotropic if $H_{n}^{T} H_{n} \neq k I$ ). In particular, the empirical correlation estimates will not yield a diagonal covariance matrix for the noise, and the assumption of independence of speech and noise will mostly not be true for short-time segments. As a result, the noise reduction that is obtained with the above estimators will not be optimal.

It is interesting to quantify the decrease in performance in such situations. Thereto we derive our so-called optimal estimator (OPT).

Assume that both the clean and noisy observation matrices $H_{s}$ and $H_{x}$ are observable (= cheating experiment). We will now explain how to find the optimal-in LS sense-gain factors $g_{\mathrm{OPT}, i}$ [20]. If the SVD of $H_{x}$ is given by

$$
H_{x}=U \Sigma V^{T},
$$

the optimal estimate $\hat{H}_{\mathrm{OPT}}$ of $H_{s}$ is defined as

$$
H_{\mathrm{OPT}}=\underset{G_{p}}{\arg \min }\left\|U_{p} \Sigma_{p} G_{p} V_{p}^{T}-H_{s}\right\|_{F}^{2}
$$

where, again, the subscript $p$ denotes truncation to the $p$ largest singular vectors/values (of $H_{x}$ ).

In other words, based on the exact knowledge of $H_{s}$, we modify the singular values of $H_{x}$ such that $H_{\mathrm{OPT}}$ is closest to $H_{s}$ in LS sense.

Based on the dyadic decomposition of the SVD, it can be shown that the optimal gains $g_{\mathrm{OPT}, i}(i=1, \ldots, p)$ are given by the following expression:

$$
G_{p, \mathrm{OPT}}=\operatorname{diag}\left\{U_{p}^{T} H_{s} V_{p}\right\} \Sigma_{p}^{-1}
$$

where $\operatorname{diag}\{A\}$ is a diagonal matrix constructed from the elements on the diagonal of matrix $A$. 
Proof. The values $g_{\mathrm{OPT}, i}(i=1, \ldots, p)$ are found by minimising the following cost function that is equivalent to (27):

$$
C\left(g_{1}, \ldots, g_{p}\right)=\sum_{k=1}^{m} \sum_{l=1}^{q}\left(H_{s}(k, l)-\sum_{j=1}^{p} g_{j} H_{x, j}(k, l)\right)^{2}
$$

where $A(k, l)$ is the element on row $k$ and column $l$ of matrix $A$, and $H_{x, j}=\sigma_{j} u_{j} v_{j}^{T}$ is the $j$ th rank-one matrix in the dyadic decomposition of $H_{x}$.

Taking the derivative of $C$ with respect to $g_{i}$ and setting to zero yield:

$$
\frac{\partial C}{\partial g_{i}}=2 \sum_{k=1}^{m} \sum_{l=1}^{q}\left(\left(H_{s}(k, l)-\sum_{j=1}^{p} g_{j} H_{x, j}(k, l)\right) H_{x, i}(k, l)\right)=0 .
$$

Since $u_{i}^{T} v_{j}=\delta_{i, j}$ and $v_{i}^{T} v_{j}=\delta_{i, j}$, we get

$$
g_{\mathrm{OPT}, i}=\frac{u_{i}^{T} H_{s} v_{i}}{\sigma_{i}}
$$

Note that in the derivation of the optimal estimator we do not take into account the averaging along the antidiagonals to extract the enhanced signal. However, the latter operation is not necessarily needed to obtain an optimal result [21].

Also, it can be proven that $g_{i, \mathrm{OPT}}=g_{i, \mathrm{MV}}$ if the assumptions of orthogonality and white noise are fulfilled [20].

\subsection{Visualisation of the gain factors}

An interesting comparison between the different estimators is obtained by plotting the gain factors $g_{i}$ as a function of the unbiased spectral SNR:

$$
\mathrm{SNR}_{\text {spec,unbiased }}=10 \log _{10} \frac{\bar{\sigma}_{i}^{2}}{\sigma_{\nu}^{2}} .
$$

By rewriting the expressions for $g_{i}$ as a function of $a \stackrel{\text { def }}{=} \bar{\sigma}_{i}^{2} / \sigma_{\nu}^{2}$, we get

$$
\begin{gathered}
g_{\mathrm{LS}, i}=1, \quad g_{\mathrm{MV}, i}=\frac{a}{1+a} \\
g_{\mathrm{SVA}, i}=\left(\frac{a}{1+a}\right)^{1 / 2}, \quad g_{\mathrm{TDC}, i}=\frac{a}{\mu+a}, \\
g_{\mathrm{SDC}_{-} 1, i=\exp \left(-\frac{\beta}{2 a}\right),} g_{\mathrm{SDC}_{-} 2, i}=\left(\frac{a}{1+a}\right)^{\gamma / 2} .
\end{gathered}
$$

In Figure 2 these gains are plotted as a function of the unbiased spectral SNR. Evidently, for all estimators, $g_{i}$ ranges from 0 (low spectral SNR, only noise) to 1 (high spectral SNR, noise free).

In practice, some of the estimators require flooring in order to avoid negative values for the weights $g_{i}$. Indeed, in these estimators the singular values $\bar{\sigma}_{i}$ of the clean-speech matrix are implicitly estimated as $\sigma_{i}^{2}-\sigma_{v}^{2}$. Evidently, the latter expression can become negative, especially in very noisy conditions. Negative weights become apparent when the gain factors are expressed (and visualised) as a function of the $b i$ ased spectral SNR spec,biased $=10 \log _{10}\left(\sigma_{i}^{2} / \sigma_{\nu}^{2}\right)$.

\subsection{Relation to spectral subtraction and Wiener filtering}

From the above discussion the strong similarity between subspace-based speech enhancement and spectral subtraction should have become clear [6]. While spectral subtraction is based on a fixed FFT, the SVD-based method relies on a data-dependent KLT, ${ }^{3}$ which results in larger computational load. For a frame of $N$ samples, the FFT requires $(N / 2) \cdot \log _{2}(N)$ operations, whereas the complexity of the SVD of a matrix with dimensions $m \times q$ is given by $\mathcal{O}\left(m q^{2}\right)$. Recall that $m \gg q$, with $q$ typically between 8 and 20 , and with $m+q-1=N$. This means that for typical values of $N$ and $q$, the SVD requires 10 up to 100 times more computations than the FFT. However, real-time implementations of subspace speech enhancement are feasible on nowadays (high-end) hardware.

Another major difference between subspace-based speech enhancement and spectral subtraction is the explicit assumption of signal order or, equivalently, a rank-deficient speech observation matrix or a rank-deficient speech correlation matrix. Note that in Wiener filtering, this rank reduction is done implicitly by the estimation of a (possibly) rank-reduced speech correlation matrix.

For completeness we mention that beside FFT-based and SVD-based speech enhancement, also a DCT-based enhancement approach is possible [22]. While the DCT provides a better energy compaction than the FFT, it is still inferior to the theoretically optimal KLT transform that is used in subspace filtering.

\section{IMPLEMENTATION ASPECTS}

In this section we discuss the choice of the most important parameters in the SVD-based noise reduction algorithm, namely the frame length $N$, the dimensions of $H_{x}$, and the dimension $p$ of the signal subspace.

\subsection{Signal subspace dimension}

In theory the dimension of the signal subspace is defined by the order of the linear signal model in (1). However, in practice the speech contents will strongly vary (e.g., voiced versus unvoiced segments) and the entire signal will never exactly obey one model. Several techniques, such as minimum description length (MDL) [23] were developed to estimate the model order. Sometimes, the order $p$ is chosen on a frameby-frame basis, and, for example, chosen as the number of positive eigenvalues of the estimate $R_{s}$ of $\bar{R}_{s}$. A rather similar strategy is to set $p$ such that the energy of the enhanced signal is as close as possible to an estimate of the clean-speech energy. This concept was introduced in [24] and is called

\footnotetext{
3 The FFT and KLT coincide if the signal observation matrix is circulant.
} 


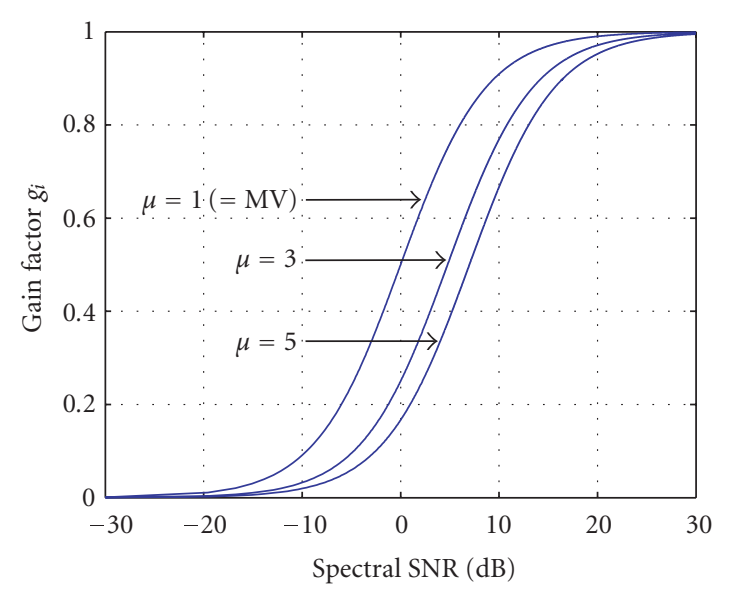

(a) TDC

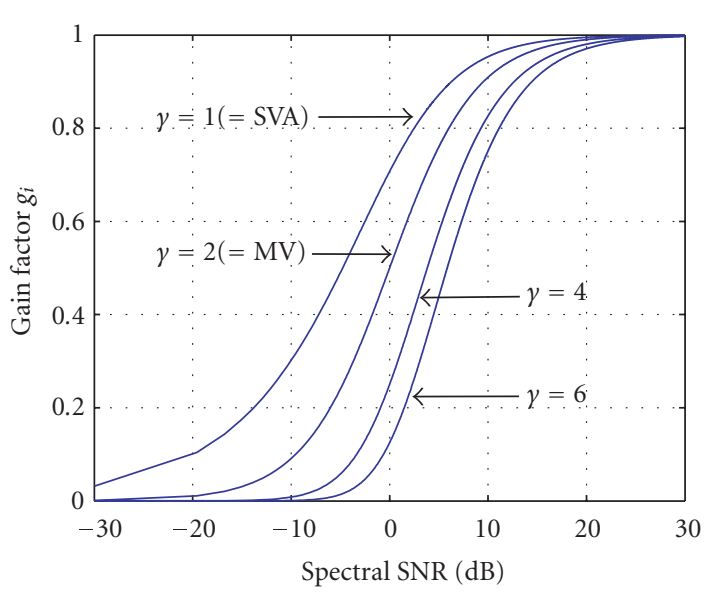

(c) SDC_2

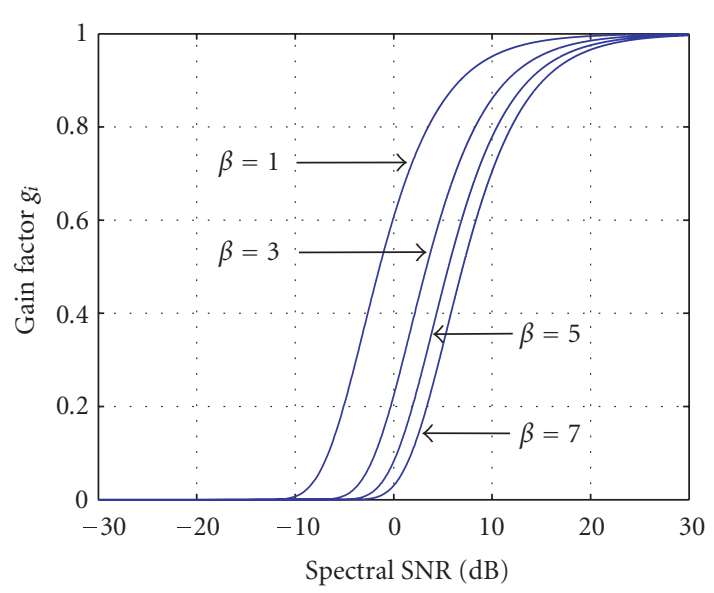

(b) SDC_1

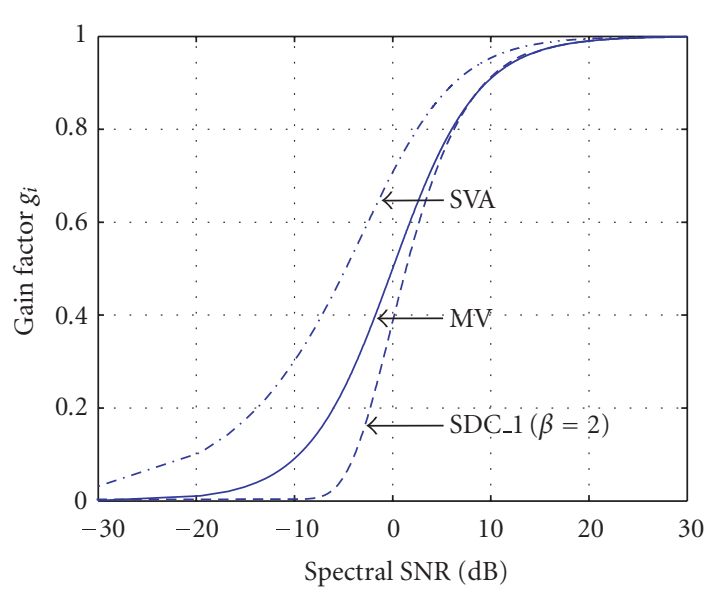

(d) MV/SVA / SDC_1

FIgURE 2: Gain factors for the different estimators as a function of the spectral SNR.

"parsimonious order". For $16 \mathrm{kHz}$ data the value of $p$ is usually around 12 .

\subsection{Frame length}

The frame length $N$ must be larger than the order of the assumed signal model, such that the correlation that is embedded in the speech signal can be fully exploited to split the latter signal from the noise. On the other hand, the frame length is limited by the time over which the speech and noise can be assumed stationary (usually 20 to 30 milliseconds). Besides, $\mathrm{N}$ must not be too large to avoid prohibitively large computations in the SVD of $H_{x}$. Hence, the value of $N$ is typically between 320 and 480 samples for $16 \mathrm{kHz}$ data.

\subsection{Matrix dimension}

Observe that the dimensions $(m \times q)$ of $H_{x}$ cannot be chosen independently due to the relation $m+q=N+1$. The smaller dimension $q$ of $H_{x}$ should be larger than the order of the assumed signal model, such that the separation into a signal and a noise subspace is possible. If $q$ is small, for example, $q \approx p$, the smallest nontrivial singular value of $H_{s}$ decreases strongly and becomes of the same magnitude as the largest singular value of the noise, such that the determination of the signal subspace becomes less accurate. For this reason, $q$ must not be taken too small [5].

A sufficiently high value for $m$ is beneficial for the noise removal, since the necessary conditions of orthogonality of speech and noise (i.e., $\left.H_{s}^{T} H_{n}=0\right)$, and white noise $\left(H_{n}^{T} H_{n}=\right.$ $\sigma_{\nu}^{2} I$ ) will on average be better fulfilled. Also, for large $m$, the noise threshold that adds up to every singular value of $H_{s}$ (see (13)) becomes more and more pronounced such that the expressions for the gain functions $g_{i}$ become more accurate. Note that the value of $m$ is bounded since the value of $q$ decreases for increasing values of $m$. A good compromise is to choose $m$ in the range 20 to 30 ( $16 \mathrm{kHz}$ data).

For more information on the choice of $m$ and $q$ we refer to $[4,5]$.

\section{EXTENSION TO COLOURED NOISE}

If the additive noise is not white, the noise correlation matrix $\bar{R}_{n}$ cannot be diagonalised by the matrix $\bar{V}$ with the right 
eigenvectors of $H_{s}$, and the expressions for the EVD of $\bar{R}_{x}(6)$ and SVD of $H_{x}(13)$ are no longer valid. In this case, a different procedure should be applied. It is assumed that the noise statistics have been estimated during noise-only segments, or even during speech activity itself [25-27]. Below, we shortly review the most common extensions of the basic subspace filtering theory to coloured noise conditions.

\subsection{Explicit pre- and dewhitening}

The modified SVD noise reduction scheme can easily be extended to the general coloured noise case if the Cholesky factor $R$ of the noise signal is known or has been estimated. ${ }^{4}$ Indeed, the noise can be prewhitened by a multiplication by $R^{-1}[4,5]$ :

$$
H_{x} R^{-1}=\left(H_{s}+H_{n}\right) R^{-1}
$$

such that

$$
\left(H_{n} R^{-1}\right)^{T}\left(H_{n} R^{-1}\right)=Q^{T} Q=I .
$$

A corresponding dewhitening operation (a postmultiplication by the matrix $R$ ) should be included after the SVD modification.

\subsection{Implicit pre- and dewhitening}

Because subsequent pre- and dewhitening can cause a loss of accuracy due to numerical instability, usually an implicit preand dewhitening is performed by working with the quotient SVD $(\text { QSVD })^{5}$ of the matrix pair $\left(H_{x}, H_{n}\right)[10]$. The QSVD of $\left(H_{x}, H_{n}\right)$ is given by

$$
\begin{aligned}
& H_{x}=\tilde{U} \Delta \Theta^{T}, \\
& H_{n}=\tilde{V} M \Theta^{T} .
\end{aligned}
$$

In this decomposition, $\tilde{U}$ and $\tilde{V}$ are unitary matrices, $\Delta$ and $M$ are diagonal matrices with $\delta_{1} \geq \delta_{2} \geq \cdots \geq \delta_{q}$ and $\mu_{1} \leq$ $\mu_{2} \leq \cdots \leq \mu_{q}$, and $\Theta$ is a nonsingular (invertible) matrix.

Including the truncation to rank $p$, the enhanced matrix is now given by [10]:

$$
\widehat{H}_{s}=\tilde{U}_{p}\left(\Delta_{p} G_{p}\right) \Theta_{p}^{T} .
$$

The expressions for $G_{p}$ are the same as for the white noise case, but considering that $\sigma_{v}^{2}$ is now equal to 1 due to the prewhitening. Also, the QSVD-based noise reduction can be interpreted as a FIR-filtering operation, in a way that is very similar to the white noise case [18].

A QSVD-based prewhitening scheme for the reduction of rank-deficient noise has recently been proposed by Hansen and Jensen [29].

\footnotetext{
${ }^{4}$ Note that $R$ can be obtained either via the $Q R$-factorisation of the noise Hankel matrix $H_{n}=Q R$, or via the Cholesky decomposition of the noise correlation matrix $R_{n}=R^{T} R$.

${ }^{5}$ Originally called the generalised SVD in [28].
}

\section{Optimal estimator}

The generalisation of the optimal estimator (OPT) in (28) to the coloured noise case is rather straightforward. The expression for the QSVD implementation is found by

$$
\hat{H}_{\mathrm{OPT}}=\underset{G_{p}}{\arg \min }\left\|\tilde{U}_{p} \Delta_{p} G_{p} \Theta_{p}^{T}-H_{s}\right\|_{F}^{2}
$$

which leads to [20]

$$
G_{p, \mathrm{OPT}}=\operatorname{diag}\left\{\tilde{U}_{p}^{T} H_{s} \Theta_{p}^{T}\right\}\left(\operatorname{diag}\left\{\Theta_{p}^{T} \Theta_{p}\right\}\right)^{-1} \Delta_{p}^{-1} .
$$

This expression is very similar to the white noise case (28), except for the inclusion of a normalisation step. The latter is necessary since the columns of the matrix $\Theta$ are not normalised.

\subsection{Signal/noise KLT}

A major drawback of pre- and dewhitening is that not only the additive noise but also the original signal is affected by the transformation matrices since

$$
H_{x} R^{-1}=H_{s} R^{-1}+H_{n} R^{-1} .
$$

The optimisation criteria (e.g., minimal signal distortion) will hence be applied to a transformed, that is, distorted, version of the speech and not to the original speech. It can be shown that in this case only an upper bound of the signal distortion is minimised when the TDC and SDC estimators are applied [30].

As a possible solution, Mittal and Phamdo [30] proposed to classify the noisy frames into speech-dominated frames and noise-dominated frames, and to apply a clean-speech KLT or noise KLT, respectively. This way, prewhitening is not needed.

\subsection{Noise projection}

The pre- and dewhitening can also be avoided by projecting the coloured noise onto the clean signal subspace [11].

Based on the estimates $R_{n}$ and $R_{x}$ of the correlation matrices $\bar{R}_{n}$ and $\bar{R}_{x}$ of the noise and noisy speech, we obtain an estimate $R_{s}$ of the clean-speech correlation matrix $\bar{R}_{s}$ as

$$
R_{s}=R_{x}-R_{n}
$$

If $R_{s}=V \Lambda V^{T}$, the energies of the noise Hankel matrix $H_{n}$ along the principal eigenvectors of $R_{s}$ (i.e., the clean signal subspace) are given by the elements of the following diagonal matrix: ${ }^{6}$

$$
\Sigma_{c, \text { proj }}^{2}=\operatorname{diag}\left\{V^{T} R_{n} V\right\} .
$$

\footnotetext{
${ }^{6}$ Note that in general $V^{T} R_{n} V$ itself will not be diagonal since the orthogonal matrix $V$ is obtained from the EVD of $R_{s}$ and hence it diagonalises $R_{s}$ but not necessarily $R_{n}$. Consequently, the noise projection method yields a (heuristic) suboptimal solution.
} 
In the weighting matrix $G_{p}$ that appears in the noise reduction scheme for white noise removal (14), the constant $\sigma_{w}^{2}$ is now replaced by the elements of $\Sigma_{c, \text { proj }}^{2}[11]$. In other words, instead of having a constant noise offset in every signal subspace direction, we now have a direction-specific noise offset due to the nonisotropic noise property.

\subsection{Latest extensions for TDC and SDC estimators}

$\mathrm{Hu}$ and Loizou [31, 32] proposed an EVD-based scheme for coloured noise removal based on a simultaneous diagonalisation of the estimates of the clean-speech and noise covariance matrices $R_{s}$ and $R_{n}$ by a nonsingular nonorthogonal matrix. This scheme incorporates implicit prewhitening, in a similar way as the QSVD approach. ${ }^{7}$ An exact solution for the TDC estimator was derived, whereas the SDC estimator is obtained as the numerical solution of the corresponding Lyaponov equation.

Lev-Ari and Ephraim extended the results obtained by $\mathrm{Hu}$ and Loizou, and derived (computationally intensive but) explicit solutions of the signal subspace approach to coloured noise removal. The derivations allow for the inclusion of flexible constraints on the residual noise, both in the time and frequency domain. These constraints can be associated to any orthogonal transformation, and hence do not have to be associated with the subspaces of the speech or noise signal. Details about this solution are beyond the scope of this paper. The reader is referred to [12].

\section{EXPERIMENTS}

In this section we first describe simulations with the SVDbased noise reduction algorithm, and analyse its performance both in terms of SNR improvement (objective quality measurement) and in terms of perceptual quality by informal listening tests (subjective evaluation). In the second section we describe the results of an extensive set of LV-CSR experiments, in which the SVD-based speech enhancement procedure is used as a preprocessing step, prior to the recognisers' feature extraction module.

\subsection{Speech quality evaluation}

\section{Objective quality improvement}

To evaluate and to compare the performance of the different subspace estimators, we carried out computer simulations and set up informal listening tests with four phonetically balanced sentences ( $f s=16 \mathrm{kHz}$ ) that are uttered by one man and one woman (two sentences each). These speech signals were artificially corrupted with white and coloured noise at different segmental SNR levels. This SNR is calculated as the average of the frame SNR (frame length = 30 milliseconds, $50 \%$ overlap). Nonspeech and low-energy

\footnotetext{
${ }^{7}$ However, note that in the QSVD approach, the noisy speech (and not the clean speech) and noise Hankel matrices are simultaneously diagonalised.
}

frames are excluded from the averaging since these frames could seriously bias the result [33, page 45].

The coloured noise is obtained as lowpass filtered white noise, $c(z)=w(z)+w\left(z^{-1}\right)$ where $w(z)$ and $c(z)$ are the Z-transforms of the white and coloured noise, respectively. In Table 1 we summarise the average results for these four sentences. The results are obtained with optimal values (obtained by an extensive set of simulations) for the different parameters of the algorithm. For coloured noise removal the QSVD algorithm was used.

For white noise, we found by experimental optimisation that choosing $\mu=1.3, \beta=2$, and $\gamma=2$ for the TDC, SDC _ 1 , and SDC _2 estimators, respectively, is a good compromise. For coloured noise, $(\mu, \beta, \gamma)=(1.3,1.5,2.1)$. The noise reference is estimated from the first 30 milliseconds of the noisy signal. The smaller dimension of $H_{x}$ is set to 20 for all estimators.

\section{(a) Subspace dimension $p$}

The value of $p$ (given in the 4 th column of Table 1 ) is dependent on the SNR and is optimised for the MV estimator but it was found that the optimal values for $p$ are almost identical for the SDC, TDC, and SVA estimators.

A totally different situation is found for the LS estimator. Due to the absence of noise reduction in the signal subspace, the performance of the LS estimator behaves very differently from all other estimators, and its performance is critically dependent on the value of $p$. Therefore, we assign a specific, SNR-dependent value for $p$ to this estimator (as indicated between brackets in the 2 nd column of Table 1 ).

The 3rd column gives the result of the LS estimator with a frame-dependent value of $p$. The value of $p$ is derived in such a way that the energy $E_{\widehat{s}_{p}}$ of the enhanced frame is as close as possible to an estimate of the clean-speech energy $\hat{E}_{s}$ :

$$
p=\underset{l}{\arg \min }\left|\hat{E}_{s}-E_{\hat{s}_{l}}\right|
$$

where $E_{\widehat{S}_{l}}$ is the energy of the enhanced frame based on the $l$ dominant singular triplets [24].

Based on the assumption of additive and uncorrelated noise, this can be rewritten as

$$
p=\underset{l}{\arg \min }\left|\hat{E}_{s}-\left(E_{x}-\widehat{E}_{n}\right)\right|
$$

Note that $p$ cannot be calculated directly but has to be found by an exhaustive search (analysis-by-synthesis). It was found that using a frame-dependent value of $p$ does not lead to significant SNR improvements for the other estimators [20]. Also note that severe frame-to-frame variability of $p$ may induce (additional) audible artefacts.

The difference in sensitivity between the LS estimator and all other estimators to changes in the value of $p$ (for a fixed matrix order $q$ ) is illustrated in Figure 3. This figure shows the segmental SNR of the enhanced signal as a function of the order $p$ for four different values of $q$, for white noise at both an SNR of $0 \mathrm{~dB}$ (dashed line) and at an SNR of $10 \mathrm{~dB}$ (solid line). For the LS estimator (a) we observe that the SNR 
TABLE 1: Segmental SNR improvements (dB) with SVD-based speech enhancement. $N=480, f_{s}=16 \mathrm{kHz}$.

\begin{tabular}{|c|c|c|c|c|c|c|c|c|c|c|}
\hline \multirow{2}{*}{$\mathrm{SNR}(\mathrm{dB})$} & \multicolumn{10}{|c|}{ White noise } \\
\hline & $\operatorname{LS}(p)$ & $\operatorname{LS}\left(\not p^{r}\right)$ & $p$ & MV & SVA & TDC & SDC_1 & SDC _ 2 & OPT & S_SUB \\
\hline 0 & $7.14(3)$ & 8.12 & 9 & 8.23 & 7.25 & 8.23 & 8.50 & 8.28 & 9.00 & 8.33 \\
\hline 5 & $5.35(4)$ & 6.21 & 9 & 6.38 & 6.03 & 6.42 & 6.39 & 6.43 & 6.82 & 6.43 \\
\hline 10 & $3.81(7)$ & 4.37 & 13 & 4.78 & 4.40 & 4.78 & 4.62 & 4.77 & 5.01 & 4.75 \\
\hline 15 & $2.66(9)$ & 2.90 & 17 & 3.47 & 3.24 & 3.50 & 3.38 & 3.47 & 3.55 & 3.42 \\
\hline 20 & $1.58(13)$ & 2.35 & 18 & 2.82 & 2.54 & 2.90 & 2.84 & 2.82 & 2.99 & 2.48 \\
\hline 25 & $0.89(15)$ & 1.78 & 19 & 2.30 & 1.85 & 2.35 & 2.30 & 2.38 & 2.59 & 2.02 \\
\hline \multirow{2}{*}{ SNR (dB) } & \multicolumn{10}{|c|}{ Coloured noise } \\
\hline & $\mathrm{LS}(p)$ & $\mathrm{LS}\left(\not p^{n}\right)$ & $p$ & MV & SVA & TDC & SDC_1 & SDC _ 2 & OPT & S_SUB \\
\hline 0 & $5.82(2)$ & 6.80 & 5 & 6.91 & 6.34 & 6.98 & 6.91 & 6.93 & 7.35 & 6.51 \\
\hline 5 & $4.13(4)$ & 4.93 & 10 & 5.22 & 4.53 & 5.22 & 5.15 & 5.22 & 5.54 & 4.74 \\
\hline 10 & $2.55(8)$ & 3.21 & 15 & 3.64 & 3.17 & 3.70 & 3.52 & 3.71 & 3.80 & 3.23 \\
\hline 15 & $1.38(11)$ & 1.75 & 18 & 2.38 & 2.12 & 2.47 & 2.31 & 2.48 & 2.55 & 2.01 \\
\hline 20 & $0.51(15)$ & 0.72 & 19 & 1.53 & 1.40 & 1.56 & 1.52 & 1.57 & 1.65 & 1.20 \\
\hline 25 & $0.20(18)$ & 0.60 & 20 & 1.08 & 0.85 & 1.09 & 1.11 & 1.11 & 1.34 & 0.73 \\
\hline
\end{tabular}

has a clear maximum and that the optimal value of $p$ depends on the noise level. For the MV estimator (b) we notice that the SNR saturates as soon as $q$ is above a given threshold.

The results presented here are for the white noise case but a very similar behaviour is found for the coloured noise case.

\section{(b) Comparison with spectral subtraction}

In the last column of Table 1 the results with some form of spectral subtraction are given. The enhanced speech spectrum is obtained by the following spectral subtraction formula:

$$
\begin{aligned}
\hat{S}(f) & =\left(\frac{\max \left(|X(f)|^{2}-\mu|\hat{N}(f)|^{2}, \beta|\hat{N}(f)|^{2}\right)}{|X(f)|^{2}}\right)^{1 / 2} X(f) \\
& =g_{s_{-} \text {sub }}(f) X(f)
\end{aligned}
$$

with control parameters $\mu$ and $\beta[6,33]$. The optimal values for these parameters are fixed to a value that is dependent on the SNR of the noisy speech: $\mu$ ranges from 1 (high SNR) to 3 (low SNR), and $\beta$ from 0.001 (low SNR) to 0.01 (high SNR).

\section{(c) Discussion}

From the table we observe the poor performance of the LS estimator with a fixed $p$. Since no noise reduction is done in the (signal + noise) subspace, the LS estimator causes (almost) no signal distortion (at least for $p$ larger than the true signal dimension), but this goes at the expense of a high residual noise level and lower SNR improvement. Working with a frame-dependent signal order $p$ is very helpful here, mainly to reduce the residual noise in noise-only signal frames. The impact of such a varying $p$ is rather low for the other estimators [20].

Apart from the LS estimator, all other estimators yield comparable results, except for the SVA estimator that performs clearly worse, also due to insufficient noise removal (see (22)). Overall, the TDC and SDC _2 estimators score best, with rather small deviations from the theoretical optimal result (OPT estimator). Also, SVD-based speech enhancement outperforms spectral subtraction.

\section{Perceptual evaluation}

Informal listening tests have revealed a clear difference in perceptual quality between speech enhanced by spectral subtraction on the one hand, and by SVD-based filtering on the other hand. While the first one introduces the well-known musical noise (even if a compensation technique like spectral flooring is performed), the latter produces a more pleasant form of residual noise (more noise-like, but less annoying in the long run). This difference is especially true for low-input SNR. The intelligibility of the enhanced speech seems to be comparable for both methods. These findings are confirmed by several other studies $[6,10]$.

Note that the implementations of subspace-based speech enhancement and spectral subtraction are very similar. While spectral subtraction is based on a fixed FFT, the SVD-based 


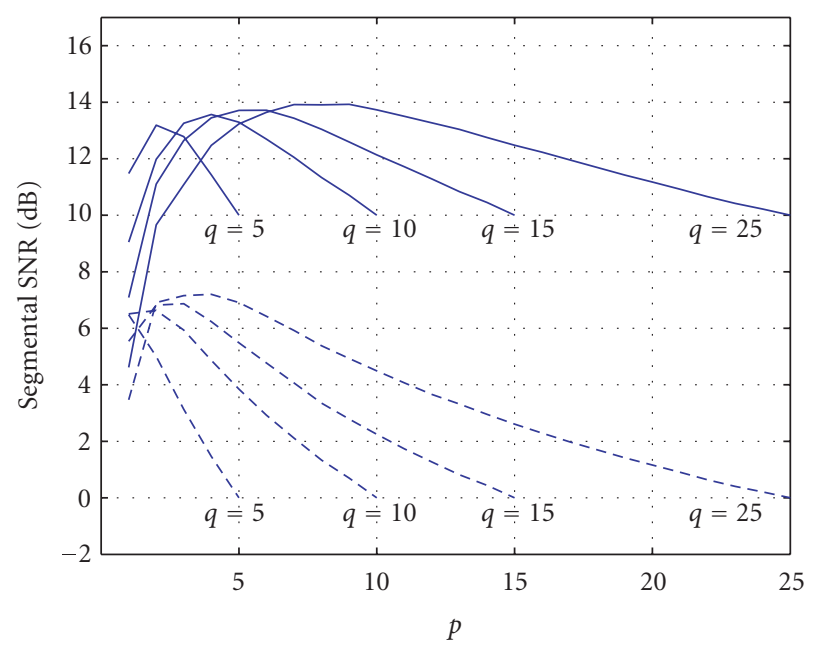

(a) LS

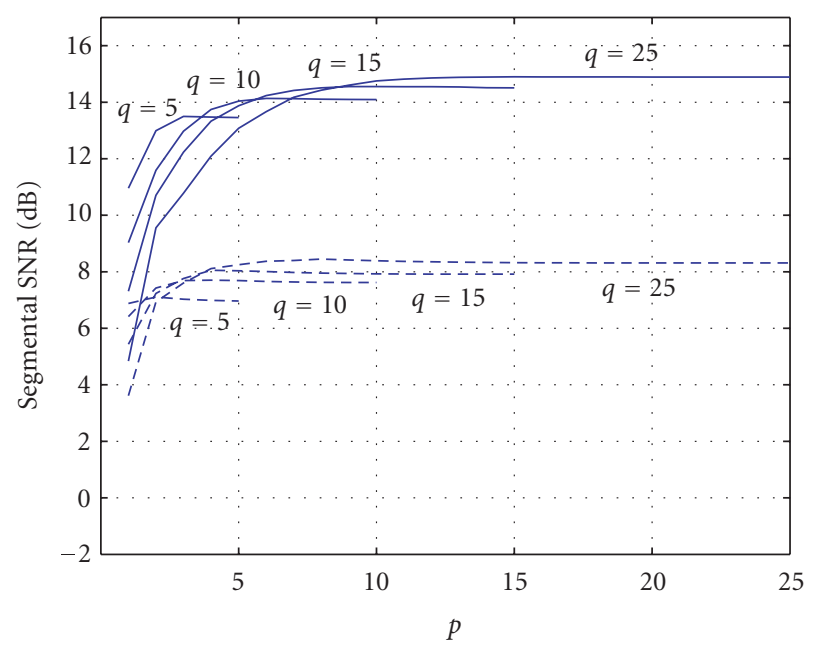

(b) MV

FIGURE 3: Segmental SNR of the enhanced signal as a function of the order $p$ of the enhanced Hankel matrix, for different values of $q$. A solid line is used for noisy speech at $10 \mathrm{~dB}$ SNR and a dashed line for $0 \mathrm{~dB}$ SNR. (a) LS estimator. (b) MV estimator (representative of all estimators that perform noise reduction in the signal subspace).

method relies on a data-dependent KLT, which results in a larger computational load.

\subsection{Speech recognition experiments}

In this section we describe the results of an extensive set of LV-CSR experiments, in which the SVD-based speech enhancement procedure is used as a preprocessing step, prior to the recognisers' feature extraction module. Experiments are carried out with all five above-mentioned estimators. The performance of SVD-based filtering will be compared to spectral subtraction.

\section{Evaluation database}

As test material we took the resource management (RM) database (available from LDC [34]). These data are considered as clean data, to which distortions were artificially added. The SNR is determined in the same way as in the Aurora 4 benchmark database [35]. The ratio of signal-to-noise energy is defined after filtering both signals with the G.712 characteristic. To determine the speech energy, the ITU recommendation P.56 is applied by using the corresponding ITU software. The noise energy is calculated as RMS value with the same software. Also here, two noise types were added to the clean speech, namely white noise and coloured noise (obtained as lowpass filtered white noise). This was done for the following set of SNR values that yield meaningful recognition accuracies: 5, 10, 15, 20, 25, and $30 \mathrm{~dB}$. In this case, a simple global SNR measure is used, since there is no evidence that ASR accuracies correlate more with a segmental than with a global SNR measure.

\section{Speech recogniser}

For the assessment of the different subspace approaches we use a speaker-independent LV-CSR system [36]. The system that we use is beneficial for this purpose because of its fast experiment turnaround time and good baseline accuracy. In the preprocessing, the common mel frequency cepstral coefficients (MFCCs) are combined with their first- and second-order derivatives, of which 25 features are selected. To remove convolutional noise distortions, a cepstral mean normalisation (CMN) step is included. The acoustic modelling is based on a set of 46 phones. Each of the 139 HMM states is modelled by a mixture of 128 tied Gaussian distributions, which are selected from a total set of 4526 Gaussians [37]. Training is performed with the original clean RM data; no retraining with SVD-enhanced speech material is conducted. A word-pair grammar language model for the $1 \mathrm{k}$ word vocabulary is used, while decoding is done with a timesynchronous beam search algorithm. The training material consists of the SI-109 train set, while testing is done with the Feb89 test set.

\section{Results}

The estimation criteria mentioned above are compared in a series of recognition experiments. First, we will present the recognition results that can be achieved with the optimal values for all parameters. Afterwards, we will discuss the influence of the most important algorithm parameters (matrix dimensions, signal subspace order).

Table 2 presents the word recognition rates (\%) for both white and coloured noise distortions. First, the reference recognition rates (i.e., without noise reduction) are given, 
TABLE 2: Word recognition accuracies (\%) with SVD-based speech enhancement-RM Feb89 test set.

\begin{tabular}{l|cccccc|ccccccc}
\hline \multirow{2}{*}{ SNR $(\mathrm{dB})$} & \multicolumn{9}{|c}{ White noise } & \multicolumn{5}{c}{ Coloured noise } \\
\cline { 2 - 14 } & 5 & 10 & 15 & 20 & 25 & 30 & 5 & 10 & 15 & 20 & 25 \\
\hline Ref & 2.30 & 4.57 & 25.07 & 52.13 & 73.45 & 85.63 & 1.91 & 12.10 & 41.62 & 67.51 & 83.16 & 90.82 \\
LS & 2.73 & 14.17 & 41.62 & 67.67 & 82.43 & 89.34 & 2.42 & 19.29 & 51.19 & 71.81 & 84.97 & 91.14 & \\
MV & 14.14 & 42.68 & 71.22 & 86.26 & 91.21 & 93.05 & 17.53 & 50.06 & 75.79 & 88.95 & 91.64 & 92.97 \\
SVA & 6.60 & 31.12 & 64.86 & 82.35 & 90.12 & 92.31 & 9.14 & 37.13 & 69.97 & 84.07 & 89.50 & 91.84 \\
TDC & 18.00 & 46.00 & 73.72 & 87.15 & 91.57 & 93.17 & 24.95 & 53.30 & 77.39 & 88.99 & 91.80 & 92.89 \\
SDC_1 & 7.77 & 38.34 & 67.24 & 83.52 & 88.64 & 90.63 & 15.50 & 42.33 & 72.20 & 86.22 & 89.54 & 89.81 \\
SDC_2 & 16.75 & 47.56 & 74.81 & 86.84 & 91.37 & 93.06 & 22.18 & 51.27 & 75.95 & 88.99 & 91.68 & 92.98 \\
OPT & 36.78 & 60.02 & 77.31 & 87.62 & 90.71 & 92.82 & 41.12 & 62.55 & 79.15 & 87.19 & 90.78 & 92.58 \\
S_SUB & 21.32 & 49.51 & 70.68 & 85.40 & 90.63 & 92.82 & 24.68 & 53.22 & 77.55 & 87.90 & 91.92 & 93.28 \\
\hline
\end{tabular}

followed by the best recognition rates for each of the estimation criteria. The recognition accuracy for the original clean data is $95.12 \%$.

The SVD-based speech enhancement is integrated in the preprocessing module of the ASR system which allows a synchronisation of speech enhancement operations and feature extraction. The analysed frames (no windowing) have a length of 30 milliseconds with 20 milliseconds overlap. On average the smaller dimension $q$ of the Hankel matrix is around 8 and-except for the LS estimator-no rank reduction of $H_{x}$ was performed, that is, $p=q$ (as will be explained below). For the TDC and SDC estimators, the best results are obtained with $\mu=3, \beta=0.8$, and $\gamma=2$.

The results with the spectral subtraction algorithm are obtained with $\beta=0.005$ ( $\approx$ optimal value at all SNRs $)$ and with $\mu$ between 2 (highest SNR) and 6 (lowest SNR).

For the optimal (OPT) estimator, the number of free parameters increases with $N$ and $q$. To allow a fair comparison with the other estimators, we took a frame length of 30 milliseconds $(N=480)$ and set $q=8$.

The clear difference in reference recognition rates between the white and coloured noise cases can mainly be explained by the way the SNR is calculated in the Aurora framework.

For the TDC and SDC estimators, the best results are obtained with $\mu=3, \beta=1$, and $\gamma=4$.

\section{(a) General observations}

From our experiments we learn that the MV, TDC, and SDC _2 estimators are most effective in increasing the recognition accuracy of noisy data. The exponential expression of the SDC $\_1$ estimator forces the smallest singular values to become very small, even for moderate values of $\beta$. This more "aggressive" noise reduction causes more signal distortion, ${ }^{8}$ which explains its rather weak performance. On the other hand, the LS estimator yields very poor results due to its

\footnotetext{
${ }^{8}$ For this reason, the parameter $\beta$ must not be set larger than 1.
}

high residual noise level. Intuitively, the results obtained with the optimal estimator (OPT) give an indication of an upper bound on the recognition accuracy improvement that could be obtained by SVD-based filtering of noisy speech data. The spectral subtraction technique leads to recognition accuracies that are comparable to those obtained by the SVD-based approach.

\section{(b) Hankel matrix dimension q}

For the LS estimator the best results are obtained with $q=8$. For higher values of $q$, the recognition rates tend to saturate, or even slightly decrease. For all other estimators (except for the optimal estimator), the choice of $p$ is not crucial and is best taken between 8 and 20, which is favourable for a limited computational complexity.

\section{(c) Subspace order $p$}

The order $p$ plays a crucial role in optimising the recognition accuracy improvement for the LS estimator. In Figure 4(a) the word recognition accuracy is plotted against the value of $p$, both for white noise at 10 (dashed line) and 20 (solid line) dB SNR. Moreover, the optimal value of $p$ strongly depends on the SNR. Hence, it is important to obtain a reliable estimate of the a priori SNR of the noisy signal. As a rule of thumb, the value of $p$ can be set approximately equal to $q / 2(\mathrm{SNR}<10 \mathrm{~dB}), 2 / 3 q(10<\mathrm{SNR}<20 \mathrm{~dB})$, and $0.8 q(\mathrm{SNR}>20 \mathrm{~dB})$. When using a variable order $p$, the speech recognition accuracy considerably drops. The most obvious explanation for this observation may be the nonstationarities that are introduced at the level of the signal distortion and the residual noise. It is well known that speech recognisers are very sensitive to variations of the background noise level, more than to the absolute level of the noise [38].

For all estimators that combine the removal of the noise subspace with the suppression of the noise in the signal subspace, a different dependency is observed. In order to obtain the best recognition rate, the value of $p$ should be set almost 


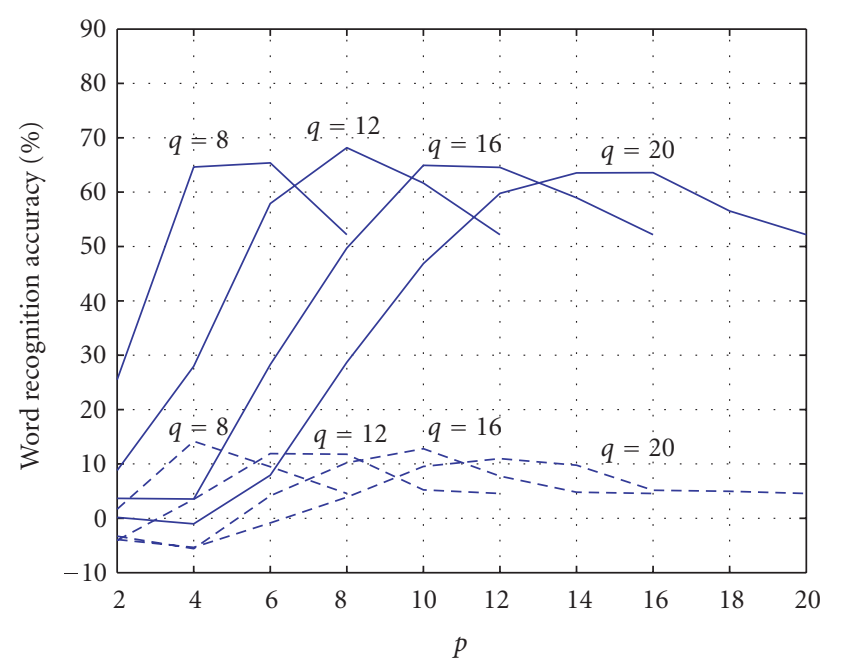

(a) LS

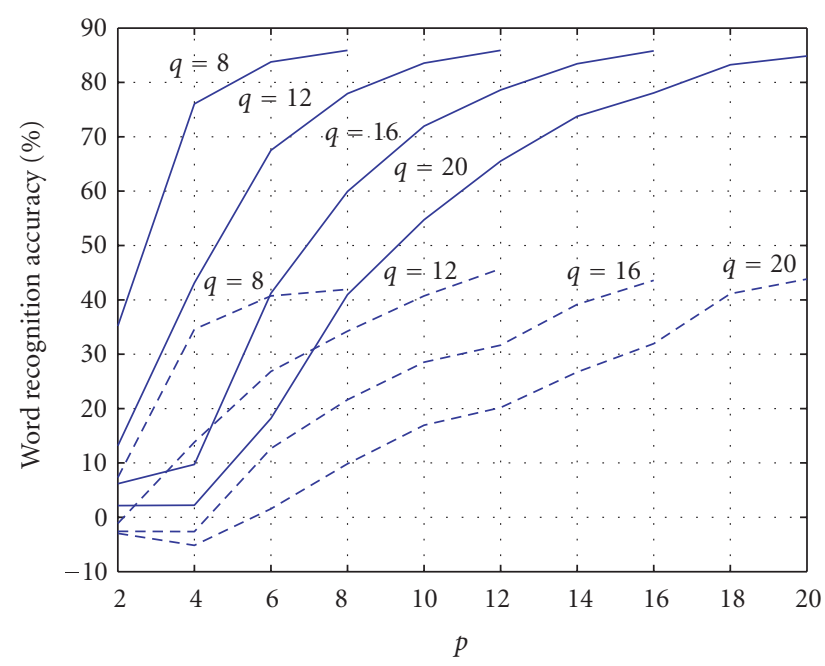

(b) MV

FIGURE 4: Word recognition accuracy for the SVD-based enhanced signal as a function of the order $p$ of the enhanced Hankel matrix, for different values of $q$. A solid line is used for noisy speech at $20 \mathrm{~dB}$ SNR and a dashed line for $10 \mathrm{~dB}$ SNR. (a) LS estimator. (b) MV estimator (representative of all estimators that perform noise reduction in the signal subspace).

equal to $q$ (no nulling of the noise subspace in this case). In general, it is observed that with increasing $p / q$, the recognition rate gradually saturates to reach its maximal value at $p \approx q$. This is illustrated in Figure 4(b) for the MV estimator. A similar behaviour is observed for the other estimators that perform noise reduction in the signal subspace. The most plausible explanation for this observation is that truncation introduces signal distortions (e.g., gaps in the spectrum of the enhanced signal) that compromise a proper decoding with the clean-speech acoustic models. Note that this observation is independent of the SNR of the input signal. Using a variable order $p$ instead of a fixed one has almost no influence on the recognition rates.

\section{CONCLUSION}

Signal subspace speech enhancement has proven to be a powerful and very flexible tool, both for increasing the speech intelligibility in speech communications applications and for improving the accuracy of automatic speech recognisers in additive noise environments. In this paper we reviewed the basic theory of subspace filtering and compared the performance of the most common optimisation criteria. We derived a theoretical estimator to experimentally assess an upper bound to the performance that can be achieved by any subspace-based method, both for the white and the coloured noise case. We called this the optimal estimator.

The simulations as well as the automatic speech recognition (ASR) experiments that were described in this paper have given a better insight in the potential of subspace-based speech enhancement techniques in general, and in the relative performance of the available estimators in particular.

It was found that KLT-based speech enhancement is to be preferred over FFT-based (i.e., spectral subtraction) algorithms, even though the latter operates at a (much) lower computational load. As described in earlier studies [6], subspace filtering produces much less musical noise than spectral subtraction does. Also, for improved speech recognition accuracy in noisy environments, SVD-based speech enhancement turned out to be highly competitive with spectral subtraction.

Overall, the MV estimator-including its generalisation to the TDC estimator-and the SDC estimator proved to give the best results. However, the difference in performance with the optimal estimator remains significantly high in the framework of robust speech recognition, which motivates further research in this respect. The experiments further showed that a truncation of the signal observation matrix (i.e., nulling of the noise subspace) is only advisable for pure speech enhancement applications but not for speech recognition.

We believe that the use of more advanced noise estimation techniques and further integration of the subspace filtering into the ASR preprocessing module will lead to improved performance.

\section{ACKNOWLEDGMENT}

The authors would like to thank Peter Karsmakers for his help in carrying out the computer simulations.

\section{REFERENCES}

[1] D. W. Tufts, R. Kumaresan, and I. Kirsteins, "Data adaptive signal estimation by singular value decomposition of a data matrix," Proceedings of the IEEE, vol. 70, no. 6, pp. 684-685, 1982.

[2] J. A. Cadzow, "Signal enhancement-a composite property mapping algorithm," IEEE Transactions on Acoustics, Speech, and Signal Processing, vol. 36, no. 1, pp. 49-62, 1988. 
[3] M. Dendrinos, S. Bakamidis, and G. Carayannis, "Speech enhancement from noise: a regenerative approach," Speech Communication, vol. 10, no. 1, pp. 45-57, 1991.

[4] B. De Moor, "The singular value decomposition and long and short spaces of noisy matrices," IEEE Transactions on Signal Processing, vol. 41, no. 9, pp. 2826-2838, 1993.

[5] S. Van Huffel, "Enhanced resolution based on minimum variance estimation and exponential data modeling," Signal Processing, vol. 33, no. 3, pp. 333-355, 1993.

[6] Y. Ephraim and H. L. Van Trees, "A signal subspace approach for speech enhancement," IEEE Transactions on Speech and Audio Processing, vol. 3, no. 4, pp. 251-266, 1995.

[7] Y. Hu and P. Loizou, "Perceptual weighting motivated subspace based speech enhancement approach," in Proceedings of International Conference on Spoken Language Processing (ICSLP '02), pp. 1797-1800, Denver, Colo, USA, September 2002.

[8] F. Jabloun and B. Champagne, "Incorporating the human hearing properties in the signal subspace approach for speech enhancement," IEEE Transactions on Speech and Audio Processing, vol. 11, no. 6, pp. 700-708, 2003.

[9] Y. Hu and P. C. Loizou, "A perceptually motivated approach for speech enhancement," IEEE Transactions on Speech and Audio Processing, vol. 11, no. 5, pp. 457-465, 2003.

[10] S. H. Jensen, P. C. Hansen, S. D. Hansen, and J. A. Sørensen, "Reduction of broad-band noise in speech by truncated QSVD," IEEE Transactions on Speech and Audio Processing, vol. 3, no. 6, pp. 439-448, 1995.

[11] A. Rezayee and S. Gazor, "An adaptive KLT approach for speech enhancement," IEEE Transactions on Speech and Audio Processing, vol. 9, no. 2, pp. 87-95, 2001.

[12] H. Lev-Ari and Y. Ephraim, "Extension of the signal subspace speech enhancement approach to colored noise," IEEE Signal Processing Letters, vol. 10, no. 4, pp. 104-106, 2003.

[13] P. S. K. Hansen, P. C. Hansen, S. D. Hansen, and J. A. Sørensen, "Experimental comparison of signal subspace based noise reduction methods," in Proceedings of IEEE International Conference on Acoustics, Speech, and Signal Processing (ICASSP '99), vol. 1, pp. 101-104, Phoenix, Ariz, USA, March 1999.

[14] J. Huang and Y. Zhao, "Energy-constrained signal subspace method for speech enhancement and recognition," IEEE Signal Processing Letters, vol. 4, no. 10, pp. 283-285, 1997.

[15] K. Hermus, W. Verhelst, and P. Wambacq, "Optimized subspace weighting for robust speech recognition in additive noise environments," in Proceedings of 6th International Conference on Spoken Language Processing (ICSLP '00), vol. 3, pp. 542-545, Beijing, China, October 2000.

[16] K. Hermus and P. Wambacq, "Assessment of signal subspace based speech enhancement for noise robust speech recognition," in Proceedings of IEEE International Conference on Acoustics, Speech, and Signal Processing (ICASSP '04), vol. 1, pp. 945948, Montreal, Quebec, Canada, May 2004.

[17] I. Dologlou and G. Carayannis, "Physical interpretation of signal reconstruction from reduced rank matrices," IEEE Transactions on Signal Processing, vol. 39, no. 7, pp. 1681-1682, 1991.

[18] P. C. Hansen and S. H. Jensen, "FIR filter representations of reduced-rank noise reduction,” IEEE Transactions on Signal Processing, vol. 46, no. 6, pp. 1737-1741, 1998.

[19] Y. Ephraim and H. L. Van Trees, "A signal subspace approach for speech enhancement," in Proceedings of IEEE International Conference on Acoustics, Speech, and Signal Processing (ICASSP '93), vol. 2, pp. 355-358, Minneapolis, Minn, USA, April 1993.

[20] K. Hermus, "Signal subspace decompositions for perceptual speech and audio processing," Ph.D. dissertation, Katholieke
Universiteit Leuven, ESAT, Leuven-Heverlee, Belgium, December 2004.

[21] S. Doclo and M. Moonen, "GSVD-based optimal filtering for single and multimicrophone speech enhancement," IEEE Transactions on Signal Processing, vol. 50, no. 9, pp. 2230-2244, 2002.

[22] I. Y. Soon, S. N. Koh, and C. K. Yeo, "Noisy speech enhancement using discrete cosine transform," Speech Communication, vol. 24, no. 3, pp. 249-257, 1998.

[23] J. Rissanen, "Modeling by shortest data description," Automatica, vol. 14, no. 5, pp. 465-471, 1978.

[24] S. Bakamidis, M. Dendrinos, and G. Carayannis, "SVD analysis by synthesis of harmonic signals," IEEE Transactions on Signal Processing, vol. 39, no. 2, pp. 472-477, 1991.

[25] R. Martin, "Noise power spectral density estimation based on optimal smoothing and minimum statistics," IEEE Transactions on Speech and Audio Processing, vol. 9, no. 5, pp. 504-512, 2001.

[26] I. Cohen, "Noise spectrum estimation in adverse environments: improved minima controlled recursive averaging," IEEE Transactions on Speech and Audio Processing, vol. 11, no. 5, pp. 466-475, 2003.

[27] S. Rangachari, P. C. Loizou, and Y. Hu, "A noise estimation algorithm with rapid adaptation for highly non-stationary environments," in Proceedings of IEEE International Conference on Acoustics, Speech, and Signal Processing (ICASSP '04), vol. 1, pp. 305-308, Montreal, Quebec, Canada, May 2004.

[28] G. Golub and C. Van Loan, Eds., Matrix Computations, Johns Hopkins University Press, Baltimore, Md, USA, 1983.

[29] P. C. Hansen and S. H. Jensen, "Prewhitening for rankdeficient noise in subspace methods for noise reduction," IEEE Transactions on Signal Processing, vol. 53, no. 10, pp. 37183726, 2005.

[30] U. Mittal and N. Phamdo, "Signal/noise KLT based approach for enhancing speech degraded by colored noise," IEEE Transactions on Speech and Audio Processing, vol. 8, no. 2, pp. 159$167,2000$.

[31] Y. Hu and P. C. Loizou, "A subspace approach for enhancing speech corrupted by colored noise," in Proceedings of IEEE International Conference on Acoustics, Speech, and Signal Processing (ICASSP '02), vol. 1, pp. 573-576, Orlando, Fla, USA, May 2002.

[32] Y. Hu and P. C. Loizou, "A generalized subspace approach for enhancing speech corrupted by colored noise," IEEE Transactions on Speech and Audio Processing, vol. 11, no. 4, pp. 334$341,2003$.

[33] G. S. Kang and L. J. Fransen, "Quality improvement of LPCprocessed noisy speech by using spectral subtraction," IEEE Transactions on Acoustics, Speech, and Signal Processing, vol. 37, no. 6, pp. 939-942, 1989.

[34] Linguistic Data Consortium (LDC), http://www.ldc.upenn. edu.

[35] H.-G. Hirsch and D. Pearce, "The AURORA experimental framework for the performance evaluation of speech recognition systems under noisy conditions," in Proceedings of International Speech Communication Association (ISCA) Workshop: Authomatic Speech Recognition: Challanges for the New Millenium (ASR '00), pp. 181-188, Paris, France, September 2000.

[36] K. Demuynck, "Extracting, modelling and combining information in speech recognition," Ph.D. dissertation, Katholieke Universiteit Leuven, ESAT, Leuven-Heverlee, Belgium, February 2001. 
[37] J. Duchateau, K. Demuynck, and D. Van Compernolle, "Fast and accurate acoustic modelling with semi-continuous HMMs," Speech Communication, vol. 24, no. 1, pp. 5-17, 1998.

[38] Y. Gong, "Speech recognition in noisy environments: a survey," Speech Communication, vol. 16, no. 3, pp. 261-291, 1995.

Kris Hermus was born in Asse, Belgium, in 1974. He received the M.S. and Ph.D. degrees in electrical engineering from the Katholieke Universiteit Leuven (Belgium) in 1997 and 2004, respectively. Currently, he is a postdoctoral research fellow of the Institute for the Promotion of Innovation by Science and Technology, Flanders (IWTFlanders) affiliated with the Speech Processing Research Group of the Electrical Engi-

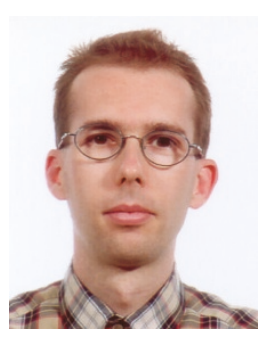
neering Department (ESAT), Katholieke Universiteit Leuven. His research interests are in the area of digital signal processing techniques for automatic speech recognition and for speech/audio modelling and coding.

Patrick Wambacq received the M.S. and Ph.D. degrees in electrical engineering from the Katholieke Universiteit Leuven (Belgium) in 1980 and 1985, respectively. From 1980 to 1998 his main interests were image processing in general, and automatic visual inspection more specifically. Since 1998, he heads the Speech Processing Research Group of the Electrical Engineering Department (ESAT), Katholieke Univer-

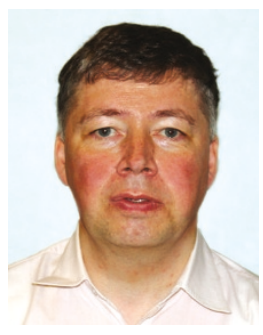
siteit Leuven, with research in the areas of robust speech recognition, spontaneous speech recognition, new architectures for recognition, speaker adaptation, clinical and educational applications of speech recognition, and speech and audio modelling.

Hugo Van hamme received the Electrical Engineering degree from the Vrije Universiteit Brussel, Belgium, in 1987, the Master's of Science degree in electrical engineering from Imperial College, London, in 1988, and the Ph.D degree from the Vrije Universiteit Brussel in 1992. He joined Lernout \& Hauspie Speech Products in 1993, where he held the positions of Researcher, Project Leader, Director, and Senior Director of Re-

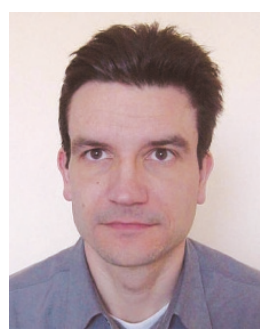
search. In 2001, he joined ScanSoft as Head of the Automotive Group. Since 2002, he has been full-time Professor at the Katholieke Universiteit Leuven. His research interests are in the areas of robust automatic speech recognition and speech processing techniques for learning applications. 